\title{
Finite Element Simulation of Viscous Fingering in Miscible Displacements at High Mobility-Ratios
}

Denis A. F. de Souza

denis@lamce.coppe.ufrj.br

\section{Alvaro L. G. A. Coutinho}

alvaro@nacad.ufrj.br

Federal University of Rio de Janeiro - UFRJ

COPPE, Dept. of Civil Engineering

PO Box 68506, Rio de Janeiro, RJ, Brazil
Numerical simulations of viscous fingering instabilities in miscible displacements at high mobility-ratios are presented. Anisotropic dispersion and monotonic viscosity profiles are considered. The coupled set of partial differential equations is approximated by the semi-discrete SUPG stabilized finite element formulation plus a discontinuity capturing technique to improve stability around the moving sharp fronts. The pressure equation is discretized by the standard Galerkin method, and a postprocessing scheme is used to improve the numerical evaluation of Darcy's velocity. In the resulting scheme all variables (concentration, pressure and velocity) are approximated by equal order linear triangular elements. A homogeneous channel and a radial system were studied. Complex nonlinear viscous fingering mechanisms for high mobility-ratio miscible displacements were observed.

Keywords: viscous fingering, miscible displacement, stabilized finite elements

\section{Introduction}

Simulation of miscible displacement flows has been widely studied in the past years due to its special importance for the petroleum industry. Enhanced oil recovery, solute transport in aquifers, packed bed regeneration and recovery of heavy oil and bitumen are examples of some applications (Homsy, 1987). Such processes involve the injection of a displacing material that is miscible with the resident fluid. The displacement of a more viscous fluid by a less viscous one leads to a mechanical instability known as viscous fingering. This instability is essentially governed by the mobility-ratio $M R$, which is defined as the ratio between the viscosity of the displaced fluid and that of the injected. However, other important factors also influence the severity of viscous fingering: heterogeneous permeability fields, gravity, anisotropic dispersion, velocity dependence of dispersion, nonmonotonic viscosity profiles, etc. High mobility-ratio displacement processes present very complex fingering patterns. Besides finger interaction mechanisms, already known for low values of mobility-ratio as shielding, spreading, tip splitting, coalescence and fading (Manickam and Homsy, 1993, 1994, 1995), other mechanisms become dominant at high mobility-ratios. These are named double coalescence, side-branching, gradual coalescence, single-sided tipsplitting, stretched coalescence, trailing lobe detachment, alternating side-branching, skewering and dense branching. As a nonNewtonian fluid is more unstable than its Newtonian counterpart, for the same low values of mobility-ratio, in miscible displacements with non-Newtonian fluids were also observed some of those complex fingering mechanisms (Azaiez and Mohamad, 2004). All of these are described by Islam and Azaiez (2005). They study high mobility-ratio miscible displacements of Newtonian fluids involving isotropic dispersion.

The miscible displacement involving incompressible fluids in a rigid porous medium can be described by different systems of equivalent equations. Many authors (Manickam and Homsy, 1993, 1995; Moissis et al., 1987; Singh and Azaiez, 2001; Tan and Homsy, 1986, 1988) consider more convenient and efficient to work in terms of vorticity and streamfunction. Coutinho and Alves (1996, 1999), Loula et al. (1999), Dias and Coutinho (2004), Coutinho et al. (2004), Juanes and Patzek (2002, 2004) employ primitive variables: pressure, velocity and concentration.

Paper accepted November, 2009. Technical Editor: Francisco Ricardo Cunha
When simulating miscible displacements a variety of methods was employed, such as finite differences, reported by Christie et al. (1991) and Waggoner et al. (1991). Other alternative has been the pseudo-spectral methods as, for example, a Hartley transform-based scheme used by Zimmerman and Homsy (1991) to simulate unstable miscible displacement. They examined the effect of anisotropic dispersion on nonlinear viscous fingering. Later Zimmerman and Homsy (1991, 1992a) extended these methods to three dimensions and performed two dimensional isotropic simulations in much broader and longer domains. Manickam and Homsy $(1994,1995)$ also used a Hartley transform based spectral method to investigate the nonlinear evolution of viscous fingering instabilities in miscible displacement flows with nonmonotonic viscosity profiles and later they studied vertical miscible displacement flows driven by both viscosity and density contrasts. Other works that employ pseudo-spectral methods are Ruith and Meiburg (2000) and Singh and Azaiez (2001). The modified method of characteristics combined with mixed finite elements is other interesting method (Moissis et al., 1987, 1988, and 1993). Other method was presented by Fast and Shelley (2004). They developed a moving overset grid scheme for simulating the dynamics of fluids interfaces. They used a thin body fitted grid that conforms to the deforming time dependent boundary and is coupled to fixed Cartesian grids. In such a way they obtained high accuracy in the interface position. Recently, Wang and Zabaras (2006) use a pairwise Markov Random Field (MRF) to model source identification in miscible displacements, obtaining accurate solutions.

Finite differences are used by most of commercial reservoir simulators; however, this method presents difficulties to handle complex geometries. Pseudo-spectral methods are highly accurate and are very efficient in the simulation of the growth of viscous fingering in porous media on parallel machines (Mangiavacchi et al., 1997). The combination of a finite element modified method of characteristics and a mixed finite element method generates a method with very little numerical dispersion, but this combined method involves different interpolation schemes for pressure, velocity and concentration.

Coutinho and Alves (1996) employed in the simulation of miscible displacements in random heterogeneous media a parallel finite element method where all variables were approximated by equal order interpolations. In this method, pressure is computed by the standard Galerkin method, and a global post-processing technique (Malta et al., 1995) is used to evaluate velocities. A parallel implementation on a distributed memory machine using global and also local post-processing techniques to compute higherorder velocity approximations is described by Loula et al. (1999). 
When solving the advection dominated concentration equation, the interest is in stability and accuracy. Stabilized finite element methods are particularly interesting for those cases. Coutinho and Alves (1996) adopted the Streamline Upwind Petrov-Galerkin (SUPG) formulation, developed by Brooks and Hughes (1982) to control spurious numerical oscillations plus a discontinuity capturing technique known as Consistent Approximated Upwind (CAU), developed by Galeão and do Carmo (1988). The resulting semi-discrete equations are approximated in time by a predictormulticorrector algorithm with variable time stepping. Coutinho et al. (2004) presented a review of the main mathematical results for the stabilized solution in both space-time and semi-discrete frameworks. Coutinho and Alves (1999) applied the formulation described above to simulate viscous fingering in miscible displacements. They studied the evolution of viscous fingering in a homogeneous media with anisotropic dispersion and also other numerical problem with a nonmonotonic viscosity profile. They used in both of cases a rectilinear geometry and the same values for the aspect ratio, $A=8$, the mobility-ratio, $M R=20$, and the global Peclet number, $P e_{G}=1000$. They showed that the formulation is able to simulate the evolution of viscous fingering in different physical situations achieving good parallel performance.

In this work, we study the ability of the formulation (Coutinho and Alves, 1996; 1999; Dias and Coutinho, 2004; Coutinho et al., 2004) described above to simulate some very high mobility-ratio displacement processes $(M R=\exp (6))$. At this time, we use two different geometries: a rectilinear Hele-Shaw cell and a radial case, where we use unstructured grids. We considered only anisotropic dispersion for both numerical examples. We observed when comparing our results with other works, Azaiez and Mohamad (2004), Islam and Azaiez (2005) and Sharon et al. (2003) that the stabilized finite element method applied here is able to represent correctly the mechanisms of viscous fingering related to the case of high mobility-ratio miscible displacements.

The remainder of this paper is organized as follows. In the next section, we briefly present the mathematical formulation adopted. Section 3 shows the semi-discrete stabilized finite element formulation for the spatial discretization of the governing equations. Also, in this section, we briefly describe the velocity postprocessing technique and the time integration scheme. Two numerical examples are analyzed in the next section. Both are miscible displacement flows with monotonic viscosity profiles at high mobility-ratios. The first one is a rectilinear Hele-Shaw cell, and the other a radial cell. The paper ends with the summary of our observations and main conclusions, in section 5 .

\section{Nomenclature}

$$
\begin{array}{ll}
A & =\text { aspect ratio, dimensionless } \\
D_{/ /} & =\text {longitudinal dispersion coefficient, dimensionless } \\
D_{\perp} & =\text { transversal dispersion coefficient, dimensionless } \\
H & =\text { characteristic width of the domain, dimensionless } \\
L & =\text { characteristic length of the domain, dimensionless } \\
M R & =\text { mobility-ratio, dimensionless } \\
P e & =\text { Peclet number, dimensionless } \\
R & =\text { constant used in the definition of the MR, dimensionless } \\
U & =\text { characteristic velocity of the fluid, dimensionless }
\end{array}
$$

\section{Greek Symbols}

$$
\begin{array}{ll}
\Omega & =\text { domain } \\
\Gamma & =\text { boundary } \\
\phi & =\text { porosity of the porous medium, dimensionless } \\
\sigma & =\text { stability parameter to the post-processed velocity } \\
& \text { dimensionless }
\end{array}
$$

$\tau \quad=$ SUPG stability parameter, dimensionless

$\eta \quad=$ penetration disturbance, dimensionless

$\zeta=$ magnitude of the concentration disturbance, dimensionless

\section{Subscripts}

// relative to the longitudinal direction or the parallel gradient direction of the solution

$\perp \quad$ relative to the transverse direction

0 relative to initial time

$e \quad$ relative to the element

c relative to the concentration magnitude

$t$ relative to post-processing

$G$ relative to global

1 relative to the Cartesian component $x$

2 relative to the Cartesian component $y$

\section{Governing Equations}

The mathematical model for the miscible displacement of one incompressible fluid by another, in a rigid porous medium, can be described by a system of partial differential equations (Peaceman, 1977). These equations in a domain $\Omega \in R^{2}$ with boundary $\Gamma$ at a time interval $[0, T]$ can be written as:

$$
\begin{aligned}
& \nabla \cdot \mathbf{v}=q \text { on } \Omega \times[0, \mathrm{~T}] \\
& \mathbf{v}=-\mathbf{A}(c) . \nabla p \text { on } \Omega \times[0, \mathrm{~T}] \\
& \phi \frac{\partial c}{\partial t}+\nabla \cdot(c \mathbf{v}-\mathbf{D}(\mathbf{v}) \cdot \nabla c)=\hat{c} q \text { on } \Omega \times[0, \mathrm{~T}]
\end{aligned}
$$

where $\mathbf{v}$ is the total Darcy velocity of the fluid mixture, $p$ its pressure, $\mathrm{c}$ its concentration and $\phi$ the porosity of the porous medium. The tensor $\mathbf{A}(\mathbf{c})$ is given as

$$
\mathbf{A}(c)=\frac{\mathbf{K}}{\mu(c)}
$$

where $\mathbf{K}$ is the position-dependent absolute permeability tensor and $\mu(c)$ is the viscosity of the fluid mixture, which depends on its concentration $c$.

The anisotropic diffusion-dispersion tensor $\mathbf{D}(\mathbf{v})$ is

$$
\mathbf{D}(\mathbf{v})=\frac{1}{|\mathbf{v}|^{2}}\left[\begin{array}{cc}
v_{1} & -v_{2} \\
v_{2} & v_{1}
\end{array}\right]\left[\begin{array}{cc}
D_{/ /} & 0 \\
0 & D_{\perp}
\end{array}\right]\left[\begin{array}{cc}
v_{1} & v_{2} \\
-v_{2} & v_{1}
\end{array}\right]
$$

where $v_{1}, v_{2}$ are the Cartesian velocity components, and $D_{/ /}$and $D_{\perp}$ are respectively the longitudinal and transverse dispersion coefficients. The wells are represented by source and sink terms denoted by $q$. The function $\hat{c}$ is specified at the sources and is equal to the resident concentration at the sinks.

We assume the usual no-flow boundary conditions

$$
\mathbf{v} \cdot \mathbf{n}=0 \text { in } \Gamma, \forall t \in[0, T]
$$

where $\mathbf{n}$ is the unit outward normal. The initial and boundary concentration conditions are known,

$$
c(\mathbf{x}, 0)=c_{0}(\mathbf{x}) \text { on } \Omega
$$




$$
\mathbf{D}(\mathbf{v}) \nabla c . \mathbf{n}=0 \quad \text { on } \quad \Gamma, \forall t \in[0, T]
$$

Uniqueness for pressure solution is ensured imposing the normalization condition,

$$
\int_{\Omega} p(\mathbf{x}, t) d \Omega=0, \quad \forall t \in[0, T]
$$

The viscosity $\mu(c)$ in Eq. (4) is assumed, as in Tan and Homsy (1992), to vary exponentially with the concentration, that is,

$$
\mu(c)=e^{-R c}
$$

where $R$ is a constant such that

$$
M R=e^{R}
$$

being $M R$ the mobility-ratio, or the ratio between the viscosities of the resident and the displacing fluids. It is important to note that when $M R>1$, nonlinear effects associated to the coupling of the equations and the convective term strongly influence stability and accuracy of numerical approximations.

\section{Finite Element Formulation}

\section{Finite Element Discretization}

In this section, we present the semi-discrete stabilized finite element formulations applied to the governing equations for miscible displacements. The semi-discrete formulation is characterized by a finite element discretization in space followed by a finite difference discretization in time. The Galerkin formulation is applied to the pressure equation and the SUPG formulation (Brooks and Hughes, 1982) plus the discontinuity-capturing operator CAU (Consistent Approximated Upwind) developed by Galeão and do Carmo (1988) is employed in the concentration equation.

We consider the space domain $\Omega$ divided in nel elements, $\Omega_{\mathrm{e}}$, $e=1,2 \ldots$ nel, where $\Omega=\bigcup_{e=1}^{n e l} \Omega_{\mathrm{e}}$ and $\Omega_{i} \cap \Omega_{j}=\varnothing$. We associate to this discretization the standard conforming set of piecewise trial and weighting finite elements spaces. Then, by substituting (2) in (1) and introducing the finite element approximation, the classical Galerkin formulation for the pressure equation is written as

$$
\int_{\Omega} \nabla w^{h} \cdot \mathbf{A}\left(c^{h}\right) \nabla p^{h} d \Omega=\int_{\Omega} w^{h} q d \Omega
$$

where $w^{h}$ is the discrete weighting function for pressure and $p^{h}$ is the discrete pressure.

The weak variational approximation for the concentration equation is written as

$$
\begin{aligned}
& \int_{\Omega} w_{c}^{h} L\left(c^{h}\right) d \Omega+ \\
& \sum_{e=1}^{n e l} \int_{\Omega^{e}} \tau \mathbf{v}^{h} \cdot \nabla w_{c}^{h} R^{e}\left(c^{h}\right) d \Omega+ \\
& \sum_{e=1}^{n e l} \int_{\Omega^{e}} \delta\left(c^{h}\right) \nabla w_{c}^{h} \cdot \nabla c^{h} d \Omega=\int_{\Omega} w_{c}^{h} \hat{c}^{h} q d \Omega
\end{aligned}
$$

where $w_{c}^{h}$ is the weighting function for concentration and $c^{h}$ is the discrete concentration. The differential operator $L\left(c^{h}\right)$ is given by

$$
L\left(c^{h}\right)=\phi \frac{\partial c^{h}}{\partial t}+\nabla \cdot\left(c^{h} \mathbf{v}^{h}-\mathbf{D}\left(\mathbf{v}^{h}\right) \nabla c^{h}\right)
$$

and the element level discrete residual is defined as,

$$
R^{e}\left(c^{h}\right)=L\left(c^{h}\right)-\hat{c}^{h} q .
$$

The first integral in Eq. (13) is the Galerkin term, the first summation of element-level integrals is the SUPG advection stabilization term and the second is the discontinuity-capturing term, needed to add stability around the moving sharp concentration fronts. In the case of advection-dominated flows with no gravity effects and using linear elements, the particular form of the SUPG parameter $\tau$ (Brooks and Hughes, 1982) is defined below:

$$
\tau=\frac{1}{2} \frac{h^{e}}{\left\|\mathbf{v}_{e}^{h}\right\|} \min \left(\frac{P e^{e}}{3}, 1.0\right)
$$

where $P e^{e}$ is the local Peclet number defined as

$$
P e^{e}=\frac{1}{2} h^{e} \frac{\left\|\mathbf{v}_{e}^{h}\right\|^{3}}{\left(\mathbf{v}_{e}^{h}\right)^{T} \mathbf{D} \mathbf{v}_{e}^{h}}
$$

being $h^{e}$ an estimate of the element size

$$
h^{e}=\sqrt{2 A^{e}}
$$

where $A^{e}$ is the element area, and $\mathbf{v}_{e}^{h}$ the velocity in the element. The form of the nonlinear diffusion parameter $\delta$ (Coutinho and Alves, 1999) in the CAU operator is as follows:

$$
\begin{aligned}
& \delta=\frac{1}{2} h^{e} \min \left(\frac{P e^{e} / /}{3}, 0.7\right) \frac{\left|R^{e}\left(c^{h}\right)\right|}{\left\|\nabla c^{h}\right\|} \\
& P e_{/ /}^{e}=\frac{1}{2} h^{e} \frac{\left\|\mathbf{v}_{e / /}^{h}\right\|^{3}}{\left(\mathbf{v}_{e / /}^{h}\right)^{T} \mathbf{D} \mathbf{v}_{e / /}^{h}} \\
& \mathbf{v}_{e / /}^{h}=\frac{\mathbf{v}_{e}^{h} \cdot \nabla c^{h}}{\left\|\nabla c^{h}\right\|^{2}} \nabla c^{h}
\end{aligned}
$$

where $P e_{/ /}^{e}$ is the local Peclet number correspondent to $\mathbf{v}_{e / /}^{h}$ which is the velocity projected in the parallel gradient direction of the solution, $\nabla c^{h}$. Note that $\delta=0$ when $\left\|\nabla c^{h}\right\|$ is zero. 


\section{Velocity Post-Processing}

When simulating miscible displacements the velocity computed directly from Darcy's law is less accurate than the other variables. Post-processing schemes may be used to obtain better approximations. Here, we adopted the global post-processing scheme of Malta et al. (1995). This scheme is based on the combination of Darcy's law variational formulation and the residue of the mass conservation equation. Given the pressure $p^{h}$ and the concentration $c^{h}$ and defining

$$
U^{h} \in\left\{\mathbf{w}^{h} \in H^{1 h}(\Omega) \times H^{1 h}(\Omega), \mathbf{w}^{h} \cdot \mathbf{n}=0 \text { in } \Gamma\right\}
$$

where $\Omega \in R^{2}$ is a domain with boundary $\Gamma$, the velocity postprocessing consists in finding $\tilde{\mathbf{v}}_{t}^{h} \in U^{h}$ such as $\forall \mathbf{w}^{h} \in U^{h}$ we have

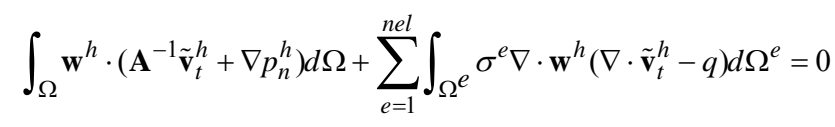

where $H^{1 h}(\Omega)$ is the usual finite elements space of finite dimension functions, the magnitude $\tilde{\mathbf{v}}_{t}^{h}$ is the post-processed velocity and

$$
\sigma^{e}=M R^{\frac{1}{2}} \tau
$$

This technique gives higher order rates of convergence for the recovered velocity, which are even better than those obtained with mixed formulations (Arbogast and Wheeler, 1995), Douglas et al. (1983). Using this approach, the problem variables, pressure, velocity and concentration are approximated by standard equal order interpolations. Stable and accurate finite element approximations are obtained for the concentration combining this post-processing technique to compute velocity with the SUPG formulation for the transport equation. It is also important to note that other alternative techniques can also be employed. Loula et al. (1999) employed both global and local post-processing techniques to compute higher-order approximations. Local post-processing is a generalization of the global post-processing based on least-squared residual of the balance equation, irrotationality condition and Darcy's law at some special points of superconvergence of the gradient. Other possibilities are, for example, a mixed stabilized finite element method with two stabilized variational formulations (Masud and Hughes, 2002). The first accommodates continuous velocity and pressure interpolations and the second accommodates continuous velocity and discontinuous pressure. Recently, Bochev et al. (2006) compare four different finite element methods for the Darcy equations and each one uses velocity and pressure approximations of the same interpolation order.

\section{Time-Marching Algorithm}

The concentration time-derivatives are approximated by the generalized trapezoidal rule (Hughes, 1987). Thus, we obtain the following block-iterative predictor-multicorrector algorithm to advance the solution in time:

- Block 1: Solve Pressure Equation

- Block 2: Compute Post-Processed Velocity Field

- Block 3: Solve Concentration Equation

The iterative process continues up to some convergence criteria is met. In this algorithm the linear system of equations corresponding to the pressure equation is solved by preconditioned conjugate gradients while that corresponding to the concentration equation is solved by the preconditioned GMRES algorithm. An element-by-element Gauss Seidel preconditioner is used in both cases. The systems of equations corresponding to the velocity postprocessing are solved using simple Jacobi iterations. Here we use variable time steps due to the strong nonlinear coupling between the pressure and concentration equations. We use an automatic timestep selection strategy based on the feedback control theory as presented in Coutinho and Alves $(1996,1999)$ and Valli et al (2005). The most time consuming step in the block-iterative scheme is solving for pressure. This usually accounts for more than half the CPU time.

\section{Numerical Experiment}

Numerical simulations of high mobility-ratio Newtonian miscible displacements in homogeneous media with anisotropic dispersion and monotonic viscosity profiles are presented. A rectilinear and a radial Hele-Shaw cell are studied.

\section{Rectilinear Hele-Shaw Cell}

We simulate a miscible displacement flow with anisotropic dispersion as investigated by Zimmerman and Homsy (1991, 1992a, 1992b) and also by Coutinho and Alves (1999). The resulting governing equations were scaled in the same manner as in the previous works. The dispersion coefficients and dimensionless parameters are also defined by Zimmerman and Homsy (2006).

The computational domain is a rectilinear Hele-Shaw cell, with an aspect ratio $A=4$ being $A=2 L / H$, where $L$ and $H$ are respectively the characteristic length and width of the computational domain. The mobility-ratio was set with a high value: $M R=$ $\exp (6.0)$, and global Peclet number, $P e_{G}=1000$, being

$$
P e_{G}=2 U L / D_{/ /}
$$

where $U$ is the characteristic velocity of the fluid. The initial condition for concentration is

$$
c_{0}(x)=\tilde{c}+\zeta f(x) \exp \left(-x^{2} / \eta^{2}\right)
$$

where $f(x)$ is a random function, $\zeta$ is a magnitude of the concentration disturbance, and $\eta$ is the penetration of the disturbance. We adopted $\zeta=0.01, \eta=L$ and,

$$
\tilde{c}=\left\{\begin{array}{cc}
1, & x=0 \\
0, & 0<x \leq L
\end{array}\right.
$$

The computational domain dimensions are: characteristic length $L=500$ and characteristic width $H=125$. This domain was discretized in $256 \times 1024$ cells, each cell subdivided into 4 triangles in a diamond pattern, generating a structured mesh with 525,569 nodes and 1,048,576 elements. We study the viscous fingering behavior at the channel inflow. The simulation time was 860 time units. At this time, the fingers had not reached the end of the channel, but this period was enough for observing the development of viscous fingering mechanisms.

We can observe here classical nonlinear interaction mechanisms as reported by Zimmerman and Homsy (1991, 1992a, 1992b) and by Coutinho and Alves (1999). These mechanisms are: shielding, where a finger that noses ahead shields the growth of the neighboring fingers; finger fading, where a finger fades in 
concentration; coalescence, where the tip of the coalescing finger bends and merges into the nearest shielding finger; pairing, produced by the action of a pairwise mechanism upon the microscopic fingers causing them to merge and tip splitting, where the streamwise directed shielding finger spreads at the tip and splits into two even fingerlets; the wider part spreads and goes through another tip-splitting mechanism, and the process continues. We may also find other more complex mechanisms observed for miscible displacements involving two non-Newtonian fluids, as described by Azaiez and Mohamad (2004), and all of them are identified by Islam and Azaiez (2005), even though they simulate high mobilityratio miscible displacement flow with isotropic dispersion. We can clearly observe in Figs. 1, 2 and 3 two different types of mechanisms: coalescence and splitting of branches. Depending on their specific features, each mechanism, within these two wide categories, adopts a suitable name. Examples of first group are: double coalescence, where two fingers adjacent to a longer finger approximate into its base and merge into it slowly; gradual coalescence, where a slightly inclined finger gradually merges into the closest one and it continues development inside of it and stretched coalescence, where a shielding finger is bordered of both sides and mixtures with the adjacent fingers. The second group contains the following mechanisms: side branching, where a shielding finger edge develops; it merges in the closest finger continuing developing inside of it; single-sided tip-splitting, where a shielding finger splits in two branches always for the same side; alternating side-branching, where a branch separates in other branches alternately for each side; skewering, where the edge of a shielding finger develops; and dense branching, where a branch separates in several branches simultaneously.

Numerical solutions for concentration are shown in Figs. 1 and 2 respectively for two different time sequences, at the simulation beginning and at an intermediate time. Figure 3 shows a configuration at the end of simulation. All figures were scaled up for better appreciating the viscous fingering mechanisms. In all Figures we provide concentration contours in the range $[0,1]$.

We observed here, besides classical viscous fingering instabilities, some of the nonlinear interaction mechanisms that appear in miscible displacements involving two non-Newtonian fluids, as reported by Azaiez and Mohamad (2004) and also in Islam and Azaiez (2005), in this case for miscible displacements at high mobility-ratio with isotropic dispersion. Thus, in Fig. 1 we can observe mechanisms as double coalescence (DC) and single-sided tip-splitting (STS). In Fig. 2, at a later time, we observe sidebranching (SB) and gradual coalescence (GC) and, in Fig. 3, we can appreciate trailing lobe detachment mechanism (TLD). We also observed in Fig. 3 that there are some branches significantly more developed than the rest. This type of behavior was also observed in non-Newtonian fluids with low values of mobility-ratio (Azaiez and Mohamad, 2004). These observations suggest that Newtonian high and non-Newtonian low mobility-ratio flow displacements develop similar instability patterns.

Here, we also show some computational data to illustrate the difficulties encountered in simulations of this type. Figures 4 and 5 present respectively the number of block iterations within each time step and the sequence of time steps produced by the automatic stepsize control algorithm. Note in Fig. 4 that 4 to 6 block iterations are needed. In the simulation beginning the time step is small, but as the solution progresses, the time steps vary orders of magnitude because of the fast development of complex fingering mechanisms, as shown in Fig. 5.
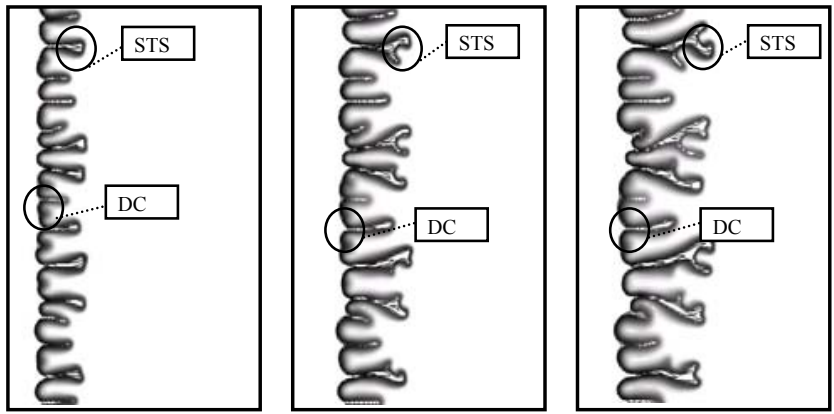

Figure 1. Concentration contours for a time sequence at the simulation beginning showing double coalescence (DC) and single-sided tip-splitting (STS) fingering mechanisms $-M R=\exp (6.0), P e_{G}=1000$ and $A=4$.
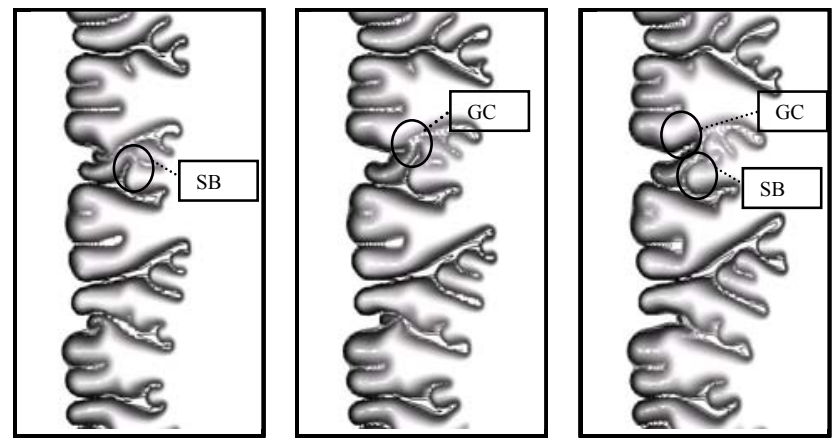

Figure 2. Concentration contours for a time sequence at an intermediate simulation time showing side-branching (SB) and gradual coalescence (GC) fingering mechanisms $-M R=\exp (6.0), P e_{G}=1000$ and $A=4$.

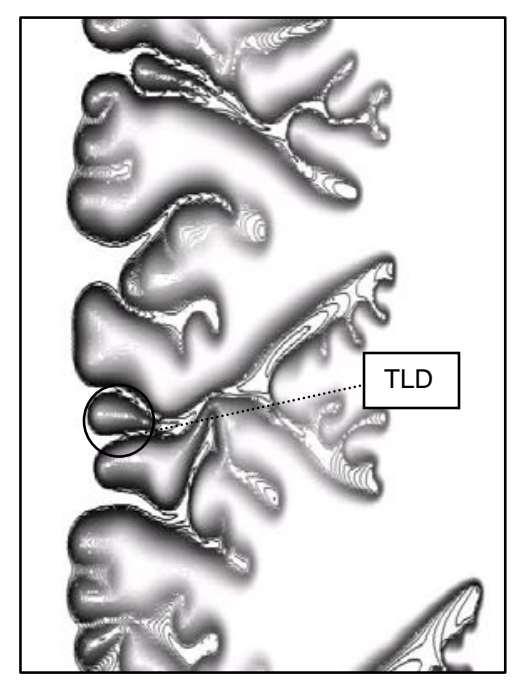

Figure 3. Concentration contours at the end of simulation showing trailing lobe detachment (TB) fingering mechanism $-M R=\exp (6.0), P e_{G}=1000$ and $A=4$. 


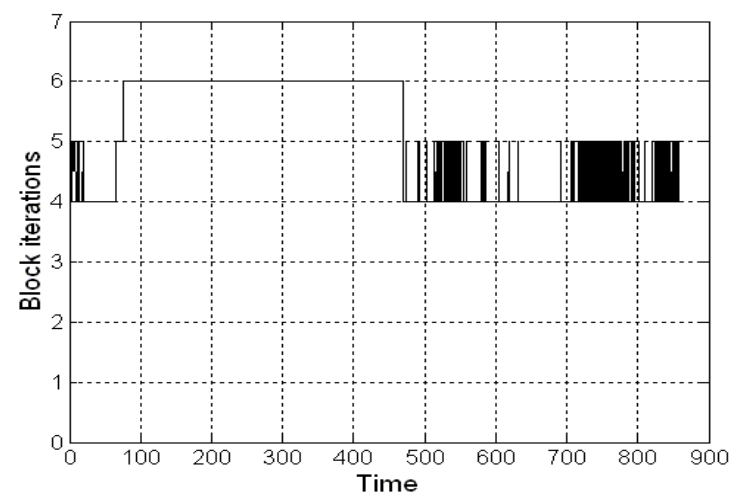

Figure 4. Non linear iterations within each time step.

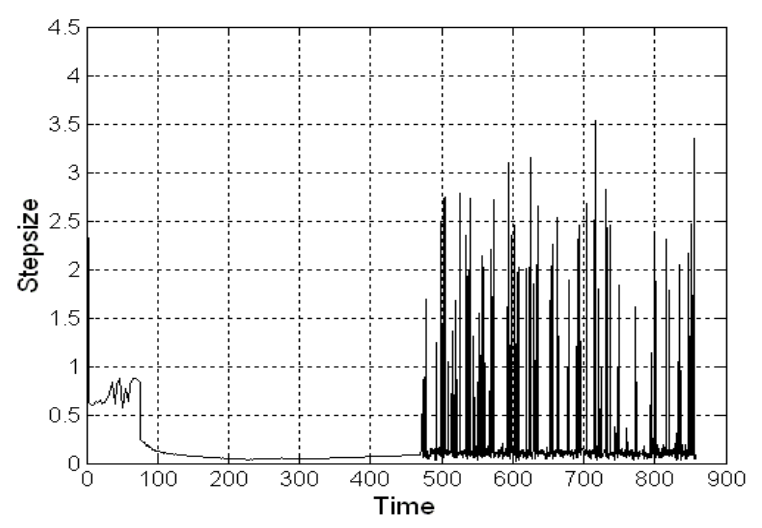

Figure 5. Time step size variation.

\section{Radial Hele-Shaw Cell}

We also analyze other miscible displacement flow with anisotropic dispersion following again Zimmerman and Homsy (1991, 1992a, 1992b). Here, the difference is that we study viscous fingering mechanisms in a radial geometry. As in the rectilinear case, $P e_{G}=1000$ and $M R=\exp (6.0)$. It was also employed a random initial condition. The computational domain is a radial configuration with internal radius $R_{\mathrm{i}}=5.0$ and external radius $R_{\mathrm{e}}=500.0$. We employ an unstructured mesh with 99,217 nodes and 197,634 elements. A mesh detail around the internal radius is shown in Fig. 6.

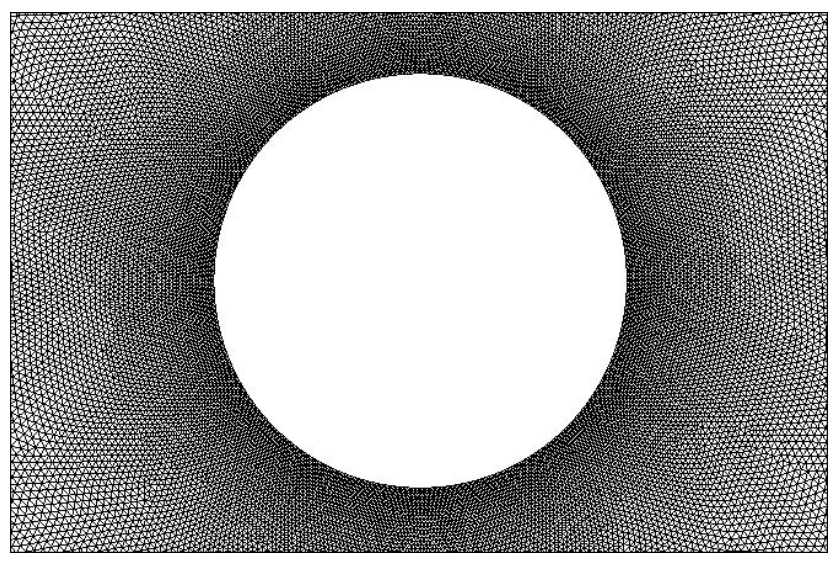

Figure 6. Finite element mesh detail around internal radius.

The initial random condition for concentration is

$$
c_{0}(\mathbf{r})=\tilde{c}+\zeta f(\mathbf{r}) \exp \left(-|\mathbf{r}|^{2} / \eta^{2}\right)
$$

where $\boldsymbol{r}$ is the position vector in polar coordinates, $f(\boldsymbol{r})$ is a random function, $\zeta$ and $\eta$ are respectively the disturbance magnitude and penetration. We adopted $\zeta=0.01, \eta=R_{e}$ and

$$
\tilde{c}=\left\{\begin{array}{cc}
1, & r=R_{i} \quad 0 \leq \theta<2 \pi \\
0, & R_{i}<r \leq R_{e} \quad 0 \leq \theta<2 \pi
\end{array}\right.
$$

The simulation time was 972 time units. We show results for respectively two different time intervals in Figs. 7 and 8: one at the simulation beginning and the other at the simulation end. Each time interval contains a sequence of three different time instants in each figure that were scaled up for better appreciating the viscous fingering features; in both figures we present concentration contours in the range $[0,1]$.

The numerical results obtained present similar behavior patterns when compared with experimental and other numerical results (Sharon et al., 2003). We also observed, as for the rectilinear case, similar viscous fingering mechanisms observed for miscible flow displacements involving two non-Newtonian fluids, as described by Azaiez and Mohamad (2004) and also in Islam and Azaiez (2005), in this case for miscible displacements at high mobility-ratio with isotropic dispersion.

We can observe mechanisms as alternating side-branching (ASB) and trailing lobe detachment (TLD) in Fig. 7. We also observed side-branching and gradual coalescence in Fig. 8.
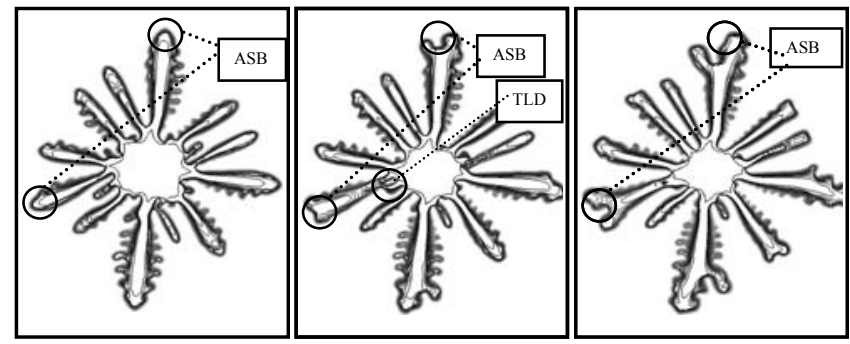

Figure 7. Concentration contours for a time sequence at the simulation beginning showing alternate side-branching (ASB) and trailing lobe detachment (TB) fingering mechanisms $-M R=\exp (6.0)$ and $P e_{G}=1000$.

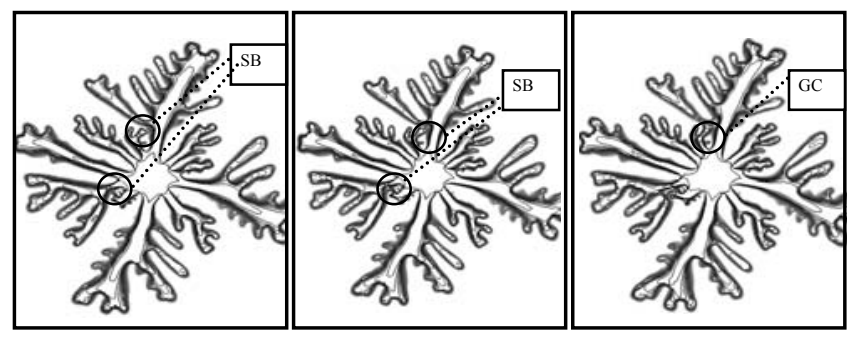

Figure 8. Concentration contours for a time sequence at the simulation end showing side-branching (SB) and gradual coalescence (GC) fingering mechanisms $-M R=\exp (6.0)$ and $P e_{G}=1000$.

Here, we also show some computational data. Figure 9 presents the sequence of time steps produced by the automatic step size control algorithm. Differently from the rectangular Hele-Shaw cell, here we note a step size increase at early simulation times and as the solution progresses and the finger mechanisms evolve, the step size diminishes. The number of block-iterations also increases in the initial steps until 6 block-iterations per step, and remains fixed until the end of the simulation. 


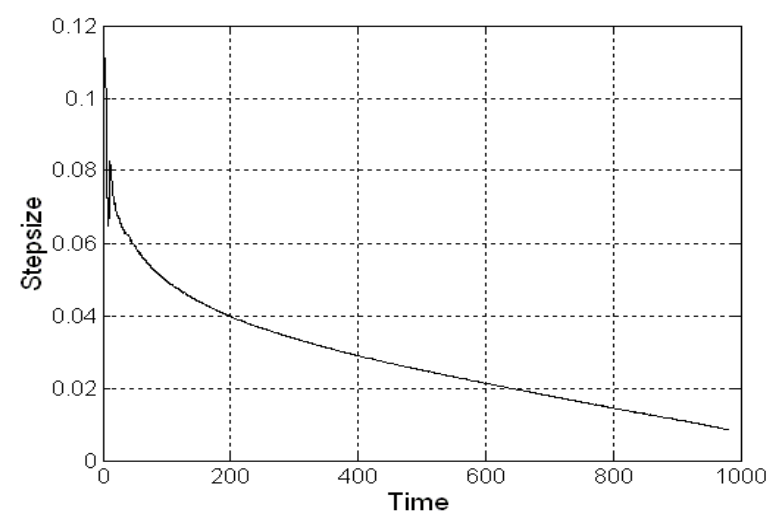

Figure 9. Time step evolution.

\section{Conclusions}

In this paper, it was presented numerical simulations to observe viscous fingering patterns in miscible displacements at high mobility-ratios. We studied numerically two different geometries: a rectilinear and a radial Hele-Shaw cell. The numerical results for both cases point out that, besides classical mechanisms of viscous fingering, other types of mechanisms become dominant at large values of mobility-ratios. They are double coalescence, sidebranching, gradual coalescence, single-sided tip-splitting, trailing lobe detachment and alternating side-branching. Some of these mechanisms, already identified in non-Newtonian fluids with low values of mobility-ratio, are reported by Azaiez and Mohamad (2004). All of them are identified by Islam and Azaiez (2005) that studied high mobility-ratio miscible displacement of Newtonian fluids involving isotropic dispersion, using a different numerical approach.

Therefore, the stabilized finite element formulation applied here was able to represent correctly the several complex mechanisms of viscous fingering related to high mobility-ratio as already observed by others in miscible displacement flows.

New stabilized formulations have been developed for Darcy flow in recent years. Some examples are space-time formulations, as in Coutinho et al. (2004), a mixed stabilized finite element method presented by Masud and Hughes (2002), four different finite element methods using velocity and pressure approximations of the same order described by Bochev et al. (2006) and multi-scale finite element formulations reported by Juanes and Patzek (2002). We want to point out that these stabilized formulations applied to complex problems, as the case of this paper, would be a great advance. In such a way we expect that the range of the mobilityratio value will be extended even more.

\section{Acknowledgements}

This work is partly supported by CNPq grant 303645/02-4. Ms Sesini is supported by CNPq grant $140680 / 02-0$, while Mr. Souza is supported by ANP grant 2001.0070-7.

\section{References}

Arbogast T., Wheeler M.F., 1995, "A characteristic-mixed finite element method for advection-dominated transport problem", SIAM J. Numer. Anal., Vol. 32, pp. 404-410.

Azaiez, J. and Mohamad, A.A., 2004, "Fingering instabilities in miscible displacement flows of non-Newtonian fluids", Journal of Porous Media, Vol. 7, pp. 29-40.
Bochev, P.B., Dohrmann, C.R., 2006, "A computational study of stabilized, low-order C0 finite element approximations of Darcy equations", Comput. Mech., Vol. 38, pp. 323-333.

Brooks, A.N. and Hughes, T.J.R., 1982, "Streamline upwind/PetrovGalerkin formulation for convection dominated flows with particular emphasis on the incompressible Navier-Stokes equations", Computer Methods in Applied Mechanics and Engineering, Vol. 32, pp. 199-259.

Christie, M.A., Muggeridge, A.H., Barley J.J., 1991, "3D simulation of viscous fingering and WAG schemes", SPE 31238, Proc. 11th SPE Symposium on Reservoir Simulation, Anaheim, CA.

Coutinho, A.L.G.A., Alves, J.L.D., 1996, "Parallel finite element simulation of miscible displacement in porous media", SPE Journal, Vol. 4, No. 1, pp. 487-500.

Coutinho, A.L.G.A., Alves, J.L.D., 1999, "Finite element simulation of nonlinear viscous fingering in miscible displacements with anisotropic dispersion and nonmonotonic viscosity profiles", Computational Mechanics, Vol. 23, pp. 108-116.

Dias, C.M., Coutinho, A.L.G.A., 2004, "Stabilized finite element methods with reduced integration techniques for miscible displacements in porous media", Int. J. Numer. Meth. Engrg.., Vol. 59, pp. 475-492.

Coutinho, A.L.G.A., Dias, C.M., Alves, J.L.D., Landau, L., Loula, A.F.D., Malta, S.M.C., Castro, R.G.S., Garcia, E.L.M., 2004, "Stabilized methods and post-processing techniques for miscible displacements", Comput. Methods Appl. Mech. Engrg., Vol. 193, pp. 1421-1436.

Douglas, J.Jr., Ewing, R.E., Wheeler, M.F., 1983, "The approximation of the pressure by a mixed-method in the simulation of miscible displacement”, R.A.I.R.O. Analyse Numer., Vol. 17, pp. 17-33.

Fast, P. and Shelley, M.J., 2004, "A moving overset grid method for interface dynamics applied to non-Newtonian Hele-Shaw flow", Journal of Computational Physics, Vol. 195, pp. 117-142.

Galeão, A.C., Carmo, E.G.D., 1988, "A consistent approximate upwind Petrov-Galerkin method for convection-dominated problems", Computer Methods in Applied Mechanics and Engineering, Vol. 68, pp. 83-95.

Homsy, G.M., 1987, "Viscous fingering in porous media". Ann. Rev. Fluid Mech., Vol. 19, pp. 271-311.

Hughes, T.J.R., 1987, “The Finite Element Method", Prentice-Hall, Englewood Cliffs.

Islam, N., Azaiez, J., 2005, "Fully implicit finite difference-pseudo spectral method for the simulation of high mobility-ratio miscible displacements", Int. J. Num. Meth. Fluids, Vol. 47, pp. 161-183.

Juanes, R., Patzek, T.W., 2002, "Multiple-scale stabilized finite elements for the simulation of tracer injections and waterflood", In: SPE/DOE $13^{\text {th }}$ Symposium on Improved Oil Recovery, Tulsa, Oklahoma, SPE 75231.

Juanes, R., Patzek, T.W., 2004, "Multiscale-stabilized finite element methods for miscible and immiscible flow in porous media", Journal of Hydraulic Research, Vol. 42, pp. 131-140 Sp. SI.

Loula, A.F.D., Garcia, A.L.M., Coutinho, A.L.G.A., 1999, "Miscible displacement simulation by finite element methods in distributed memory machines", Comput. Methods Appl. Engrg., Vol. 174, pp. 339-354.

Malta, S.M.C., Loula, A.F.D., Garcia, A.L.M, 1995, “A post-processing technique to approximate the velocity filed in miscible displacement simulations", Matemática Contemporânea, Vol. 8, pp. 239-268.

Mangiavacchi, N., Coutinho, A.L.G.A. and Ebecken, N.F.F., 1997, "Parallel pseudo-spectral simulations of nonlinear viscous fingering in miscible displacements", In: "Offshore Engineering", edited by FLLB Carneiro et al., Computational Mechanics Publications, Southampton, UK, pp. 497-506.

Manickam, O., Homsy, G.M., 1993, "Stability of miscible displacements in porous media with nonmonotonic viscosity profiles", Phys. Fluids A, Vol. 5, pp. 1356-1367.

Manickam, O., Homsy, G.M., 1994, "Simulation of viscous fingering in miscible displacements with nonmonotonic viscosity profiles", Phys. Fluids, Vol. 6, pp. 95-107.

Manickam, O., Homsy, G.M., 1995, "Fingering instabilities in vertical miscible displacements flows in porous media", J. Fluid Mech., Vol. 288, pp. 75-102.

Masud, A., Hughes, T.J.R., 2002, "A stabilized mixed finite element method for Darcy flow", Comput. Methods Appl. Mech. Engrg., Vol. 191, pp. 4341-4370.

Moissis, D.E., Miller, C.A., Wheeler, M.F., 1987, “A parametric study of viscous fingering". In: "Numerical Simulation in Oil Recovery", edited by M.F. Wheeler, Springer-Verlag, New York, pp. 227-249.

Moissis, D.E., Miller, C.A., Wheeler, M.F., 1988, "A parametric study of viscous fingering in miscible displacement by numerical simulation", Numerical Simulation in Oil Recovery, Vol. 11, pp. 227-247. 
Moissis, D.E., Wheeler, M.F., Miller, C.A., 1993, "Simulation of miscible displacements viscous fingering using a modified method of characteristics: effects of gravity and heterogeneity", SPE Reservoir Advanced Technology Series, Vol. 1, pp. 62-72.

Peaceman, D., 1977, "Fundamentals of Numerical Reservoir Simulation", Elsevier, Amsterdam.

Ruith, M., Meiburg, E., 2000, "Miscible rectilinear displacements with gravity override, part 1, homogeneous porous medium", J. Fluid Mech., Vol. 420, pp. 225-257.

Sharon, E., Moore, M.G., McCormick, W.D. and Swinney, H.L., 2003, "Coarsening of fractal viscous fingering patterns", Phys Rev Lett, Vol. 91, pp. 205-504.

Singh, B., Azaiez, J., 2001, "Numerical simulation of viscous fingering of shear-thinning fluids", Can. J. Chem. Engg., Vol. 79, pp. 961-967.

Tan, C.T., Homsy, G.M., 1986, "Stability of miscible displacements in porous media: rectilinear flow", Phys. Fluids, Vol. 29, pp. 39-45.

Tan, C.T., Homsy, G.M., 1988, "Simulation of nonlinear viscous fingering in miscible displacement", Phys. Fluids, Vol. 31, No. 6, pp. 1330-1338.

Tan, C.T., Homsy, G.M., 1992, "Viscous fingering with permeability heterogeneity”, Phys. Fluids A, Vol. 4, No. 6, pp. 1099-1101.
Valli, A.M.P, Carey, G.F., Coutinho, A.L.G.A., 2005, “Control strategies for timestep selection in finite element simulation of incompressible flows and coupled reaction-convection-diffusion processes", International Journal for Numerical Methods in Fluids, Vol. 47, No. 3, pp. 201-231.

Waggoner, J.R., Castillo, J.L., Lake, L.W., 1991, "Simulation of EOR processes in stochastically generated permeable media", Proc. $11^{\text {th }} \mathrm{SPE}$ Symposium on Reservoir Simulation, Anaheim, CA, 351-60.

Wang, J., Zabaras, N., 2006, "A Markov random field model of contamination source identification in porous media flow", International Journal of Heat and Mass Transfer, Vol. 49, No. 5-6, pp. 939-950.

Zimmerman, W.B., Homsy, G.M., 1991, "Nonlinear viscous fingering in miscible displacements with anisotropic dispersion", Phys. Fluids A, Vol. 3, pp. 1859-1872.

Zimmerman, W.B., Homsy, G.M., 1992a, "Three-dimensional viscous fingering: a numerical study", Phys. Fluids A, Vol. 4, pp. 1901-1914.

Zimmerman, W.B., Homsy, G.M., 1992b, "Viscous fingering in miscible displacements: unification of effects of viscosity contrast, anisotropic dispersion, and velocity dependence of dispersion on nonlinear finger propagation", Phys. Fluids A, Vol. 4, pp. 2348-2359. 